\title{
Trasplante renal de donantes con criterios expandidos en la región Caribe colombiana
}

\author{
Andrés Estupiñán-Bohórquez¹, Jorge Acosta-Reyes², Diego Viasus-Pérez ${ }^{3}$, Andrea García-López ${ }^{4 *}$, \\ Nasly Patino-Jaramillo 4 y Fernando Girón-Luque ${ }^{5}$ \\ ${ }^{1}$ Coordinación de donación de órganos y tejidos, Fundonar Colombia-Universidad del Norte, Barranquilla; ${ }^{2}$ Servicio de Epidemiología, Universidad \\ del Norte, Barranquilla; ${ }^{3}$ Doctorado de Enfermedades infecciosas en Trasplante, Universidad del Norte, Barranquilla; ${ }^{4}$ Departamento de Investigación, \\ Colombiana de Trasplantes, Bogotá DC; ${ }^{5}$ Cirugía de trasplantes y Departamento de Investigación, Colombiana de Trasplantes, Bogotá DC. Colombia
}

\section{Resumen}

Introducción: El uso de donantes de criterios expandidos (DCE) es una alternativa para suplir la necesidad existente de donantes de órganos. Objetivo: Evaluar los principales desenlaces a un año de los trasplantados renales con DCE en la región Caribe colombiana. Metodología: Cohorte retrospectiva de pacientes trasplantados entre 2013 y 2018. Se compararon los grupos de DCE y donantes de criterios estándar (DCS) y su asociación a los principales desenlaces. Se analizó la supervivencia del injerto y del paciente por medio del método de Kaplan-Meier. Resultados: 78 pacientes fueron incluidos, de estos, 57 eran receptores de DCS y 21 receptores de DCE. El rechazo celular agudo se presentó en el 46.2\% ( $n=36$ ), siendo el 52.4\% en trasplantados con DCE (riesgo relativo [RR]: 1.19; intervalo de confianza del 95\% [IC 95\%]: 0.72-1.97). La frecuencia de reingreso hospitalario fue del 45.1\%, la complicación más frecuente fue la infección de vías urinarias, siendo el $28.6 \%$ en DCE. Once pacientes perdieron el injerto para una supervivencia del injerto del $84.9 \%$. La supervivencia del injerto en el grupo de DCS fue del 90.7\% (IC 95\%: 79.0-96.0), comparada con el $69.2 \%$ en el grupo de DCE (IC 95\%: 43.8-84.8). Conclusión: Se encontró una diferencia significativa en la supervivencia del injerto de trasplantados renales con DCE y DCS.

Palabras clave: Trasplante de riñón. Donante de criterios expandidos. Donante de criterios estándar. Supervivencia del injerto.

\section{Kidney transplantation with expanded criteria donors in the Colombian Caribbean region}

\section{Abstract}

Introduction: The use of Expanded Criteria Donors (ECD) is an alternative to meet the existing need for organ donors. Objective: to evaluate the main outcomes of renal transplant recipients with ECD at one year in the Colombian Caribbean region. Methods: Retrospective cohort of transplant recipients between 2013 and 2018. The groups of ECD and Standard Criteria Donors (SCD) were compared and their association with the main outcomes were determine. Graft and patient survival were analyzed using the Kaplan-Meier method. Results: 78 patients were included, of these, 57 were recipients with

\section{Correspondencia:}

*Andrea García-Lopez

E-mail: aegarcia@ colombianadetrasplantes.com
Fecha de recepción: 06-07-2021

Fecha de aceptación: 12-10-2021

DOI: $10.24875 /$ NEFRO.21000028
Disponible en internet: 27-12-2021

Nefro Latinoam. 2021;18:119-127 www.nefrologialatinoamericana.com

licencia CC BY-NC-ND (http://creativecommons.org/licenses/by-nc-nd/4.0/). 
Nefro Latinoam. 2021;18

SCD and 21 with ECD. Acute cellular rejection occurred in $46.2 \%(n=36)$, among $52.4 \%$ were in patients with ECD (RR $1.1995 \% \mathrm{Cl}$ 0.72-1.97). The frequency of hospital readmission was $45.1 \%$, the most frequent complication was urinary tract infection, being $28.6 \%$ in ECD. Eleven patients had graft loss with a graft survival of $84.9 \%$. Graft survival in the SCD group was $90.7 \%$ (IC 95\% 79.0-96.0) compared with 69.2\% in the ECD group (IC 95\% 43.8-84.8). Conclusion: Significant difference was found in the graft survival of renal transplant patients with DCE and DCS.

Key words: Kidney transplant. Expanded criteria donor. Standard criteria donor. Graft survival.

\section{Introducción}

En Colombia entre 2017 y 2018 se reportaron 1,479,733 personas con enfermedad renal crónica (ERC) total de casos incidentes y prevalentes, lo que representa una prevalencia ajustada por la edad de 2.9 casos por cada 100 habitantes; de estas, 40,464 tuvieron alguna terapia de reemplazo renal. La mayor prevalencia de ERC en estadios avanzados se observó en la región Caribe. De hecho, la ciudad de Santa Marta reportó la segunda prevalencia de ERC por cada 100 habitantes ajustada por edad más elevada en el país $(4.8 ; n=18,665)$, como también Barranquilla (3.3; $n=40,912)$ y Cartagena $(4.0 ; n=38,660)^{1}$.

La ERC tiene distintas modalidades de tratamiento condicionadas por las comorbilidades del paciente y el estadio de presentación; la diálisis y el trasplante renal (TR) están reservados para las etapas finales de la enfermedad ${ }^{2}$. Al demostrar históricamente mejores resultados y mayores beneficios frente a otras opciones de tratamiento (en términos de supervivencia, calidad de vida, salud mental y funcionalidad social, entre otras), el TR constituye la terapia de elección en la persona con $\mathrm{ERC}^{3-6}$. A nivel nacional, el TR tiene una prevalencia de 147.4 personas por cada millón de habitantes ${ }^{1,7}$.

El acceso a TR está limitado por la necesidad del donante que puede ser cadavérico (fallecido por muerte cerebral o por asistolia) o vivo. Dada la escasez mundial de donantes y la necesidad aumentada de realización de trasplantes por elevación de la ERC ha sido útil el aprovechamiento de riñones provenientes de donantes de criterios expandidos (DCE) ${ }^{8}$.

Los riñones provenientes de DCE se caracterizan teóricamente por menor reserva funcional y capacidad regenerativa en comparación a los provenientes de donantes de criterios estándar (DCS $)^{9}$, lo que se ha vinculado, posterior al trasplante, con menor supervivencia a largo plazo y con mayor probabilidad de insuficiencia primaria, función retardada del injerto y episodios de rechazo agudo ${ }^{10}$. Una revisión sistemática encontró que pacientes de 40 años o más, con un tiempo de espera considerable por un riñón para trasplante, muestran una mejor supervivencia al recibir un riñón DCE que al permanecer en terapia de diálisis $^{11}$. Un estudio colombiano que analizó trasplantados con DCE entre 2007 y 2015 encontró que los pacientes trasplantados con DCE tienen una buena función del injerto renal a tres años, con supervivencia del injerto y del paciente similar a la reportada con DCS ${ }^{12}$.

En Colombia, por legislación, solo es posible la obtención de riñones de provenientes de donantes cadavéricos diagnosticados con muerte encefálica (no se rescatan riñones de fallecidos en asistolia). La región Caribe colombiana no es ajena a la escasez de los donantes, siendo históricamente la región con menor tasa de donación a nivel nacional ${ }^{13}$, pero con una necesidad aumentada de realización de trasplantes, por lo cual ha sido útil y necesario el aprovechamiento de riñones provenientes de $\mathrm{DCE}^{1}$.

Actualmente se desconoce la caracterización de los donantes renales en la región Caribe colombiana y no se ha generado evidencia clínica con respecto a los resultados a corto y mediano plazo de los receptores sometidos a TR provenientes de donantes de DCE. Si bien existen factores inmodificables relacionados con los donantes y los receptores, es necesaria una investigación comparativa que permita determinar las características diferenciales en los resultados de supervivencia de los injertos y pacientes trasplantados con riñones provenientes de DCE vs. DCS, es importante identificar aquellos factores susceptibles de intervención y así optimizar el resultado de los injertos y los pacientes trasplantados. Por lo tanto, el objetivo de este estudio fue evaluar los principales desenlaces a mediano plazo de los receptores sometidos a TR con DCE en la región Caribe colombiana.

\section{Métodos}

\section{Diseño y población}

Estudio observacional analítico de cohorte retrospectiva que incluye pacientes trasplantados por Colombiana de Trasplantes (CT) entre los años 2013 y 2018. La población de estudio incluyó a todos los pacientes 
mayores de 18 años receptores de TR de donante cadavérico provenientes de la Regional 5 de la Red Nacional de Donación y Trasplantes (Guajira, Magdalena, Bolívar, Atlántico y Sucre). No se requirió muestreo y no hubo exclusiones. Los datos fueron obtenidos de fuentes secundarias a partir de la historia clínica electrónica de los receptores de trasplante de CT, en donde se hace un seguimiento estricto mensual de los trasplantados por medio del servicio de consulta externa y/o hospitalización según el requerimiento del paciente y de los registros del formato del donante de la Regional 5. La información se digitalizó en una base de datos sistematizada y detallada hasta el nivel individual, desarrollada de manera exclusiva para este estudio.

\section{Esquema de inducción e inmunosupresión}

Todos los pacientes recibieron terapia de inducción con alemtuzumab, basiliximab o inmunoglobulina antitimocítica de acuerdo con el riesgo inmunitario y las guías médicas de trasplante. Todos los pacientes recibieron una dosis fija de metilprednisolona perioperatoriamente durante tres días con una transición a prednisona oral de dosis fija desde el día 4 al día 7 en el periodo posoperatorio. Se retiraron los esteroides una semana después del trasplante. La inmunosupresión crónica consiste en una terapia basada en inhibidores de calcineurina y antimetabolitos. Los pacientes son monitoreados de cerca en las primeras cuatro semanas posteriores al trasplante y, a partir de entonces, regresan para un seguimiento mensual. Las pruebas de histocompatibilidad realizadas en nuestro centro corresponden a antígeno leucocitario humano $(\mathrm{HLA})$, panel reactivo de anticuerpos (PRA) I y II, prueba cruzada por citometría de flujo y titulación de anticuerpos anti-HLA (este último solo en donante vivo cuando la prueba cruzada es positiva).

\section{Desenlaces}

Los desenlaces de interés son la supervivencia del injerto y del receptor a un año postrasplante. La pérdida del injerto renal se definió como el retorno a diálisis permanente documentado en la historia clínica. El rechazo agudo se definió como rechazo celular agudo documentado en una biopsia del injerto renal dentro del primer año postrasplante según la clasificación de Banff ${ }^{14,15}$. En el periodo de estudio no se presentaron rechazos mediados por anticuerpos o rechazos mixtos, por lo cual no se documentaron para este análisis. Las biopsias renales se solicitaron con indicación clínica cuando se documentó elevación de la creatinina sérica mayor o igual al $20 \%$ con respecto a la basal. Nuestro centro no realiza biopsias por protocolo.

El tratamiento del rechazo celular agudo se inició una vez confirmado el diagnóstico histológico de la siguiente manera: metilprednisolona $500 \mathrm{mg}$. IV/día en infusión durante tres días; prednisolona oral a partir del cuarto día a dosis de $0.5 \mathrm{mg} / \mathrm{kg} / \mathrm{día}$ repartido en dos tomas y durante dos semanas. Completadas las dos semanas se hace disminución semanal de $10 \mathrm{mg} /$ día hasta llegar a la dosis que recibía antes del episodio de rechazo. Se hace control de creatinina a los cinco días después de terminar los bolos. Se define respuesta al tratamiento con corticosteroides con descenso de creatinina mayor e igual al $20 \%$ de la creatinina basal del paciente.

\section{Definición de criterios expandidos}

Se definieron dos grupos de comparación de acuerdo con la presencia o no de criterios expandidos en el donante. Se definió la presencia de criterios expandidos como aquellos donantes de 60 años o mayores o de más de 50 años con al menos dos de las siguientes condiciones: historia de hipertensión arterial, creatinina sérica $>1.5 \mathrm{mg} / \mathrm{dl}$ o causa de muerte cardiovascular ${ }^{8}$. Los demás donantes que no cumplían estos criterios fueron clasificados como el grupo de criterios estándar. No realizamos biopsias preimplante de forma sistemática. La decisión de tomar o no los órganos provenientes de DCE se hace mediante la valoración macroscópica del injerto y en caso de que se requiera se envía a evaluación histológica. Los índices como KDPI/KDRI (Kidney donor profile index/Kidney donor risk profile) no son tenidos en cuenta para tomar o asignar órganos. La asignación de órganos se hace según los criterios de asignación para TR en Colombia ${ }^{16}$.

\section{Análisis estadístico}

Para caracterizar los pacientes y donantes se utilizaron medidas de resumen según correspondiera con la naturaleza y distribución de la variable. Para la comparación entre los grupos y evaluar los desenlaces se usó la prueba de chi cuadrada para las variables categóricas y la prueba de $t$ de Student o $U$ de Mann-Whitney para las variables cuantitativas según correspondía.

Se analizó la supervivencia global del injerto y del paciente mediante el método de probabilidad de supervivencia de Kaplan-Meier. El tiempo correspondió al tiempo en meses desde la fecha del trasplante hasta la pérdida del injerto, muerte o censura. Se estimaron mediana y error estándar de la función de 
supervivencia con su respectivo intervalo de confianza del 95\% (IC 95\%). Se compararon las funciones de supervivencia entre las categorías de variables sociodemográficas y clínicas mediante la prueba de Log rank.

No se realizó análisis multivariante, dado el reducido tamaño de la muestra.

\section{Aspectos éticos}

La investigación fue aprobada por el comité de ética, fueron usadas fuentes de información secundarias. No fue necesario el uso de consentimiento informado teniendo en cuenta el tipo de investigación realizada.

\section{Resultados}

\section{Características de los receptores}

Setenta y ocho pacientes fueron trasplantados en el periodo de estudio, de los cuales 21 fueron de DCE (26.9\%). El 53.8\% fueron hombres, la media de edad fue de $42.95 \pm 12.49$ años. En la mayoría de los pacientes no se conocía la etiología de la ERC. La etiología conocida más frecuente fue la glomerular. En el $69.2 \%$ de los pacientes la terapia de reemplazo renal antes del trasplante era hemodiálisis. El $7.7 \%$ de los trasplantados recibió un segundo trasplante y el tiempo promedio en lista de espera fue $21.8 \pm 17.2$ meses. La media de isquemia fría fue de $19.72 \pm 5.46$ horas. En siete receptores no se obtuvo la totalidad de la información de interés (Tabla 1).

\section{Características de los donantes}

En total hubo 45 donantes para 78 receptores de los cuales 24 eran mujeres, con una edad media de 45.04 \pm 15.0 años con un índice de masa corporal (IMC) promedio de $25.6 \pm 3.1 \mathrm{~kg} / \mathrm{m}^{2}$; se presentó hipertensión arterial (HTA) en un $28.8 \%$ de los donantes. El evento cerebrovascular fue la principal causa de muerte (66.6\%); se usó inotrópicos en un $93.3 \%$ de los donantes. El valor de la última creatinina fue en promedio 0.9 $\pm 0.2 \mathrm{mg} / \mathrm{dl}$. En cinco donantes faltó información con respecto a una variable de interés (Tabla 2).

\section{Desenlaces clínicos}

\section{SUPERVIVENCIA DEL INJERTO Y DEL PACIENTE}

En total 11 pacientes tuvieron pérdida del injerto durante el primer año de seguimiento, para una

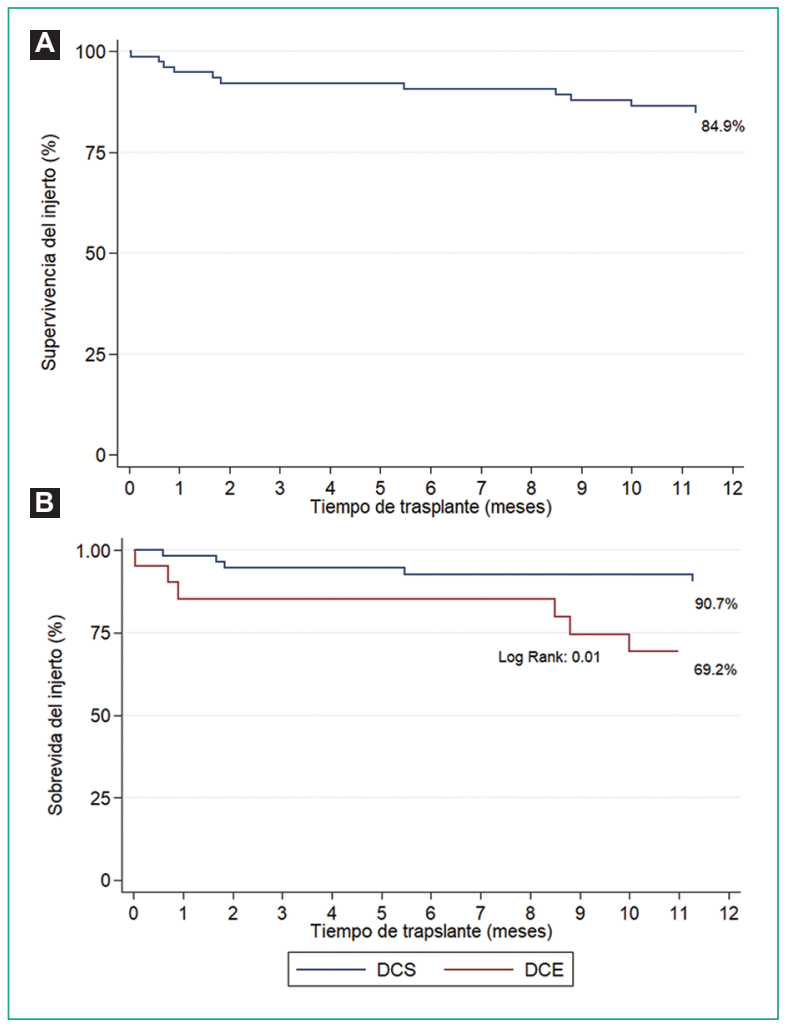

Figura 1. A: supervivencia global del injerto en receptores de trasplante renal de donante cadavérico. B: supervivencia del injerto en receptores de trasplante renal comparando los grupos de donante de criterios estándar (DCS) y donante de criterios expandidos (DCE).

supervivencia global del injerto del $84.9 \%$. Del total de pérdidas hubo cinco en el grupo de DCS con una supervivencia del $90.7 \%$ (IC 95\%: 79.0-96.0) y seis en el grupo de DCE con una supervivencia del $69.2 \%$ (IC 95\%: 43.8-84.8) (Log rank: 0.01) (Fig. 1). Las causas más frecuentes de pérdida del injerto fueron inmunitarias $(n=2)$, pielonefritis $(n=3)$ y trombosis arterial $(n=2)$.

La mortalidad al año de seguimiento ocurrió en seis pacientes. La supervivencia global del paciente fue del 91.7\% (IC 95\%: 82.4-96.2), siendo del 95.0\% (IC 95\%: 69.4-99.2) para el grupo de DCE y del $90.8 \%$ (IC 95\%: 79.3-96.0) para el grupo de DCS (Fig. 2).

\section{RECHAZO AGUDO}

El rechazo agudo al año de seguimiento se presentó en 36 trasplantados (46.2\%), siendo para DCE del $52.4 \%$ y para DCS del $43.9 \%$, con aumento del riesgo en un $19.4 \%$ para los receptores de DCE, sin 
Tabla 1. Características generales de los receptores de trasplante renal en la cohorte

\begin{tabular}{|c|c|c|c|}
\hline \multicolumn{4}{|c|}{ Características generales de los receptores de trasplante renal en la cohorte } \\
\hline Características de los receptores & Total $(\mathbf{n}=78)$ & DCS $(n=57)$ & DCE $(n=21)$ \\
\hline Edad (años), media DE & $42.9(12.49)$ & $39.5(11.74)$ & $52.2(9.51)$ \\
\hline $\begin{array}{l}\text { Sexo, n (\%) } \\
\text { Masculino } \\
\text { Femenino }\end{array}$ & $\begin{array}{l}42(53.8) \\
36(46.2)\end{array}$ & $\begin{array}{l}30(52.6) \\
27(47.4)\end{array}$ & $\begin{array}{c}12(57.1) \\
9(42.9)\end{array}$ \\
\hline IMC $\left(\mathrm{kg} / \mathrm{m}^{2}\right)$, media, DE & $26.7(4.26)$ & $26.7(4.21)$ & $26.7(4.48)$ \\
\hline $\begin{array}{l}\text { Etiología de la insuficiencia renal, n (\%) } \\
\text { Desconocida } \\
\text { HTA } \\
\text { Congénita } \\
\text { Glomerular } \\
\text { Diabetes } \\
\text { Obstructiva } \\
\text { Otra }\end{array}$ & $\begin{array}{c}40(51.3) \\
3(3.8) \\
6(7.7) \\
17(21.8) \\
7(9) \\
4(5.1) \\
1(1.3)\end{array}$ & $\begin{aligned} 30 & (52.6) \\
2 & (5.3) \\
5 & (8.8) \\
15 & (26.3) \\
1 & (1.8) \\
3 & (5.3) \\
1 & (1.8)\end{aligned}$ & $\begin{array}{c}10(47.6) \\
1(4.8) \\
1(4.8) \\
2(9.5) \\
6(28.6) \\
1(4.8) \\
0\end{array}$ \\
\hline $\begin{array}{l}\text { Tipo de diálisis recibida, n (\%) } \\
\text { Prediálisis } \\
\text { Hemodiálisis } \\
\text { Peritoneal }\end{array}$ & $\begin{array}{c}7(9) \\
54(69.2) \\
17(21.8)\end{array}$ & $\begin{array}{c}4(7) \\
43(75.4) \\
10(17.5)\end{array}$ & $\begin{array}{c}3(14.3) \\
11(52.4) \\
7(33.3)\end{array}$ \\
\hline Tiempo en diálisis (meses) ${ }^{*}$, media, DE & $51.7(43.19)$ & $54.4(42.55)$ & $43.8(45.32)$ \\
\hline Tiempo en lista de espera (meses), media, DE & $21.8(17.20)$ & $22.1(17.36)$ & $20.8(17.14)$ \\
\hline $\begin{array}{l}\text { Mismatch, n (\%) } \\
1 \\
2 \\
3 \\
4 \\
5 \\
6\end{array}$ & $\begin{array}{c}1(1.3) \\
1(1.3) \\
8(10.39) \\
19(24.68) \\
29(37.6) \\
19(26.6)\end{array}$ & $\begin{array}{c}0 \\
1(1.75) \\
7(12.28) \\
14(24.56) \\
20(35.0) \\
15(26.3)\end{array}$ & $\begin{array}{c}1(5.0) \\
0 \\
1(5.0) \\
5(25.0) \\
9(45.0) \\
4(20.0)\end{array}$ \\
\hline Trasplante previo, n (\%) & $6(7.7)$ & $4(7)$ & $2(9.5)$ \\
\hline $\begin{array}{l}\text { Tipo de inducción, n (\%) } \\
\text { Alemtuzumab } \\
\text { Basiliximab } \\
\text { Globulina de conejo }\end{array}$ & $\begin{array}{c}1(1.3) \\
12(15.3) \\
65(83.3)\end{array}$ & $\begin{array}{c}1(1.7) \\
8(14.0) \\
48(84.2)\end{array}$ & $\begin{array}{c}0(0) \\
4(19.0) \\
17(80.9)\end{array}$ \\
\hline PRA I Positivo, n (\%) & $8(10.2)$ & $7(12.2)$ & $1(4.7)$ \\
\hline PRA II Positivo, n (\%) & $9(11.5)$ & $8(14.0)$ & $1(4.7)$ \\
\hline Tiempo de isquemia fría (horas), media, DE & $19.7(5.46)$ & $20.1(0.70)$ & $18.6(1.28)$ \\
\hline
\end{tabular}

*En la variable tiempo en diálisis se tuvieron 7 datos perdidos.

${ }^{\dagger}$ No realizamos anticuerpo específico de donante como parte de la caracterización de riesgo inmunitario.

IMC: índice de masa corporal; HTA: hipertensión arterial; DE: desviación estándar; DCS: donantes de criterios estándar; DCE: donantes de criterios expandidos; PRA: panel reactivo de anticuerpos.

que esto implique una diferencia estadísticamente significativa (riesgo relativo [RR]: 1.19; IC 95\%: 0.72-1.97).

\section{REINGRESO HOSPITALARIO}

En general los pacientes receptores de DCE tuvieron menor frecuencia de reingreso hospitalario comparado con los de DCS, sin encontrar una diferencia estadísticamente significativa (RR: 0.90; IC 95\%: (0.51-1.59)

\section{COMPLICACIONES}

La complicación reportada más frecuente fue la infección de vías urinarias y se presentó en un total de 17 pacientes. Aunque la proporción de complicaciones 
Nefro Latinoam. 2021;18

Tabla 2. Características generales de los donantes de riñón

\begin{tabular}{|c|c|c|c|}
\hline \multicolumn{4}{|c|}{ Características generales de los donantes de riñón } \\
\hline Características de los donantes & Total $(n=45)$ & DCS $(n=57)$ & DCE $(n=21)$ \\
\hline Edad (años), media DE & $45(15.0)$ & $39.1(13.0)$ & $61(5.4)$ \\
\hline $\begin{array}{l}\text { Sexo, } n(\%) \\
\text { Masculino } \\
\text { Femenino }\end{array}$ & $\begin{array}{l}21(46.6) \\
24(53.3)\end{array}$ & $\begin{array}{l}15(45.4) \\
18(54.5)\end{array}$ & $\begin{array}{l}6(50) \\
6(50)\end{array}$ \\
\hline IMC $\left(\mathrm{kg} / \mathrm{m}^{2}\right)$, media DE & $25.6(3.1)$ & $25.2(3.3)$ & $26.5(2.4)$ \\
\hline $\begin{array}{l}\text { Grupo sanguíneo, n (\%) } \\
\text { A } \\
\text { B } \\
\text { AB } \\
0\end{array}$ & $\begin{array}{c}14(31.1) \\
6(13.3) \\
1(2.2) \\
24(53.3)\end{array}$ & $\begin{array}{c}10(30.3) \\
4(12.1) \\
1(3.0) \\
18(54.5)\end{array}$ & $\begin{array}{l}4(33.3) \\
2(16.6) \\
0(0) \\
6(50)\end{array}$ \\
\hline $\begin{array}{l}\text { Comorbilidades, n (\%) } \\
\text { HTA } \\
\text { DM } \\
\text { Alcoholismo }\end{array}$ & $\begin{array}{c}13(28.8) \\
5(11.1) \\
2(4.4)\end{array}$ & $\begin{array}{l}5(15.1) \\
2(6.0) \\
1(3.0)\end{array}$ & $\begin{array}{c}8(66.6) \\
3(25.0) \\
1(8.3)\end{array}$ \\
\hline $\begin{array}{l}\text { En la última hospitalización, n (\%) } \\
\text { Infección } \\
\text { Fiebre } \\
\text { Secreciones bronquiales* }\end{array}$ & $\begin{array}{c}5(11.1) \\
5(11.1) \\
13(28.8)\end{array}$ & $\begin{array}{l}4(12.1) \\
5(15.1) \\
5(15.1)\end{array}$ & $\begin{array}{c}1(8.3) \\
0(0) \\
8(66.6)\end{array}$ \\
\hline $\begin{array}{l}\text { Causa de muerte, n (\%) } \\
\text { Evento cerebrovascular } \\
\text { Trauma craneoencefálico }\end{array}$ & $\begin{array}{l}30(66.6) \\
15(33.3)\end{array}$ & $\begin{array}{l}20(60.6) \\
13(39.3)\end{array}$ & $\begin{array}{c}10(83.3) \\
2(16.6)\end{array}$ \\
\hline Última creatinina $(\mathrm{mg} / \mathrm{dl})$, media, DE & $0.9(0.2)$ & $0.8(0.2)$ & $0.9(0.2)$ \\
\hline Mantenimiento con inotrópicos, $\mathrm{n}(\%)$ & $42(93.3)$ & 31 (93.9) & $11(91.6)$ \\
\hline
\end{tabular}

fue más alta en los receptores de DCE, tampoco se encontró una diferencia estadísticamente significativa (RR: 1.36; IC 95\%: 0.77-2.40).

En la tabla 3 se describen los principales desenlaces clínicos en los receptores de TR, comparando los grupos de DCS y DCE.

\section{Discusión}

En la comunidad internacional el uso de riñones de DCE es aún controvertido, pues se asocia con mayores complicaciones, costos y se pueden encontrar más pérdidas del injerto en este grupo de receptores ${ }^{17}$. Sin embargo, también se ha visto que la mortalidad de los receptores de DCE es mucho menor a la de los pacientes con ERC que no son trasplantados y que podrían ser candidatos a recibir un injerto ${ }^{18}$, por lo que tener DCE amplía la posibilidad de TR. Esta investigación permitió conocer la evaluación del TR en la región
Caribe colombiana comparativamente entre injertos DCE y DCS a un año de seguimiento entre 2013 y 2018.

La medición de la supervivencia del injerto es el método más adecuado y usado para evaluar los resultados después del TR, otras mediciones importantes son la función del injerto (que suele medirse mediante la concentración plasmática de creatinina), la supervivencia del paciente, el número de episodios de rechazo, los días de hospitalización y los índices de calidad de vida. Con frecuencia se evalúan las supervivencias al cabo de 1, 5 y 10 años. La pérdida a corto plazo del injerto se presenta en los primeros 12 meses donde las causas de pérdida más frecuentes son las complicaciones técnicas y el rechazo ${ }^{19}$. Pasado el año la incidencia es menor y generalmente estable a lo largo del tiempo 20,21 .

La supervivencia global del injerto para este estudio fue del $84.9 \%$, cifra que es comparable a un estudio 
Tabla 3. Desenlaces clínicos en los receptores de trasplante renal de donante cadavérico

\begin{tabular}{|c|c|c|c|c|}
\hline \multicolumn{5}{|c|}{ Desenlaces clínicos en los receptores de trasplante renal de donante cadavérico } \\
\hline Variable & Total $(\mathbf{n}=\mathbf{7 8})$ & DCS $(n=57)$ & DCE $(n=21)$ & Valor de p \\
\hline Rechazo celular agudo, $\mathrm{n}(\%)^{*}$ & $36(46.2)$ & $25(43.9)$ & $11(52.4)$ & 0.503 \\
\hline Reingreso hospitalario, $\mathrm{n}(\%)$ & $36(45.15)$ & $27(47.36)$ & $9(42.85)$ & 0.256 \\
\hline $\begin{array}{l}\text { Complicaciones, n (\%) } \\
\text { Hematoma } \\
\text { Fístula urinaria } \\
\text { Infección del sitio operatorio } \\
\text { Infección urinaria }\end{array}$ & $\begin{aligned} 30 & (38.4) \\
2 & (2.6) \\
7 & (9.0) \\
4 & (5.1) \\
17 & (21.8)\end{aligned}$ & $\begin{aligned} 20 & (35.0) \\
2 & (3.5) \\
5 & (8.8) \\
2 & (3.5) \\
11 & (19.3)\end{aligned}$ & $\begin{array}{c}10(47.6) \\
0 \\
2(9.5) \\
2(9.5) \\
6(28.6)\end{array}$ & 0.455 \\
\hline Pérdida del injerto, $\mathrm{n}(\%)$ & $11(14.1)$ & $5(8.7)$ & $6(28.5)$ & 0.032 \\
\hline $\begin{array}{l}\text { Causa pérdida del injerto, n (\%) } \\
\text { Inmunitario } \\
\text { Pielonefritis } \\
\text { Trombosis arterial } \\
\text { Otra }\end{array}$ & $\begin{array}{l}2(2.56) \\
3(3.84) \\
2(2.56) \\
4(5.12)\end{array}$ & $\begin{array}{c}0 \\
2(2.57) \\
0 \\
3(5.26)\end{array}$ & $\begin{array}{c}2(9.52) \\
1(4.7) \\
2(9.52) \\
1(4.73)\end{array}$ & 0.186 \\
\hline $\begin{array}{l}\text { Mortalidad, } \mathrm{n}(\%) \\
\text { Con injerto funcionante } \\
\text { Con injerto no funcionante }\end{array}$ & $\begin{array}{l}6(7.6) \\
5(6.4) \\
1(1.2)\end{array}$ & $\begin{array}{l}5(8.7) \\
5(8.7) \\
1(1.7)\end{array}$ & $\begin{array}{l}1(4.7) \\
0(0) \\
0(0)\end{array}$ & 0.161 \\
\hline
\end{tabular}

*Todos los rechazos fueron de tipo celular. No se documentaron rechazos humorales o mixtos.

DE: desviación estándar; DCS: donantes de criterios estándar; DCE: donantes de criterios expandidos.

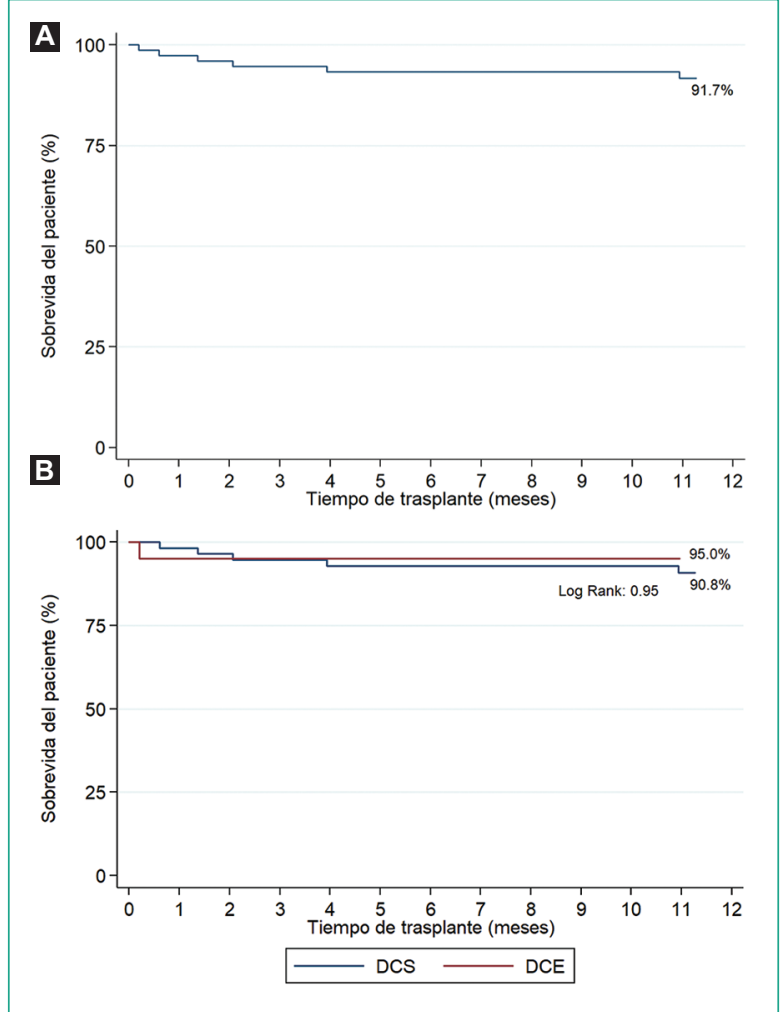

Figura 2. A: supervivencia global del paciente en receptores de trasplante renal de donante cadavérico. B: supervivencia del paciente en receptores de trasplante renal comparando los grupos de donante de criterios estándar (DCS) y donante de criterios expandidos (DCE). previo realizado en nuestra institución en donde reportaron supervivencia del $94 \%$ en el primer año, para todas las sedes de Colombia ${ }^{22}$. Sin embargo, la supervivencia varía ampliamente entre los países de Latinoamérica, encontrándose cifras entre el 73 y el $94 \%$, siendo el $82-87 \%$ para Chile $^{23}$, el $84-94 \%$ para Méxi$\mathrm{Co}^{24}$ y el $73-84 \%$ para Brasil ${ }^{25}$. Para esta cohorte, los datos mostraron mayor incidencia de pérdida del injerto en los receptores de DCE, lo cual está acorde con lo reportado en otros estudios internacionales donde Ios DCE tienen mayor riesgo de pérdida del injerto al año de seguimiento ${ }^{11,26}$. El análisis de supervivencia del injerto renal al año mostró que hubo diferencias estadísticamente significativas entre los grupos, lo cual se considera similar a lo reportado en Valjalo et al. ${ }^{10}$.

Dado que solo hubo un evento de mortalidad en el grupo de receptores con DCE, el poco tamaño de muestra limita la realización de asociaciones para este evento. Los datos obtenidos demuestran un bajo porcentaje de mortalidad de los trasplantados al año del procedimiento quirúrgico. Cabe resaltar que un trasplantado pudo haber fallecido sin que esto represente necesariamente la falla del injerto, es decir, pudo morir con injerto funcionante.

En esta cohorte se encontró una alta frecuencia de rechazo agudo si se compara con otras investigaciones que dan cuenta del rechazo agudo a nivel mundial, las 
cuales han determinado, por lo general, un mayor grado de rechazo en los DCE, comprendido entre el 5 y el $20 \%^{26-32}$. Una de las posibles explicaciones es que antes del 2018 nuestras guías de manejo indicaban iniciar la terapia inmunosupresora con inhibidores de la calcineurina una vez el receptor tuviera creatinina sérica menor o igual a $3 \mathrm{mg} / \mathrm{dl}$ por su posible nefrotoxicidad. En la actualidad, la terapia inmunosupresora se inicia en el postoperatorio inmediato, con lo cual la frecuencia de rechazos se ha disminuido a alrededor del $16 \%$. Otro posible factor es que mantenemos un protocolo de inmunosupresión libre de esteroides y esto puede contribuir a tener una frecuencia de rechazos un poco más alta que en los centros que no son libres de esteroides. El rechazo agudo desfavorece los resultados tanto en el injerto como en el paciente ${ }^{33}$. Cabe destacar que el rechazo no se asocia únicamente al tipo del donante, sino que hay otras variables que pueden explicarlo.

Algunas limitaciones de este estudio incluyen la naturaleza retrospectiva, el tamaño de la muestra y que los datos provienen de un solo centro de trasplante, lo cual limita la generalización de los resultados a otros contextos

Entre las fortalezas encontramos que este es el estudio con mayor proporción de DCE reportado en Colombia, es el primero en reportar desenlaces en TR en la región Caribe de Colombia y la revisión exhaustiva de la información, por lo que hubo pocas pérdidas.

\section{Conclusiones}

Los pacientes que recibieron un TR con DCE tienen menor supervivencia del injerto a los que recibieron un injerto con criterios estándar sin tener un impacto significativo en la mortalidad. No obstante, la reducida supervivencia del injerto no significa una falta de beneficio terapéutico. En contraste, Ios DCE son una alternativa demostrada ampliamente frente al beneficio en supervivencia obtenido al comparar con los pacientes que permanecen en lista de espera. Paralelamente, se deben realizar esfuerzos adicionales y exhaustivos para seleccionar los receptores adecuados y así maximizar la utilidad de esta estrategia para disminuir la mortalidad en lista de espera. En Colombia no existen estudios previos que comparen desenlaces entre estos grupos de donantes, por lo cual se considera que estos resultados pueden incentivar la evaluación de DCE como estrategia para aumentar el número de TR.

\section{Financiamiento}

Este estudio no tuvo ningún tipo de patrocinio.

\section{Conflicto de intereses}

Los autores declaran no tener ninguna relación financiera o personal que pudieran dar lugar a conflicto de intereses.

\section{Responsabilidades éticas}

Protección de personas y animales. Los autores declaran que para esta investigación no se han realizado experimentos en seres humanos ni en animales.

Confidencialidad de los datos. Los autores declaran que en este artículo no aparecen datos de pacientes.

Derecho a la privacidad y consentimiento informado. Los autores declaran que en este artículo no aparecen datos de pacientes.

\section{Bibliografía}

1. Cuenta de alto costo. Situación de la enfermedad renal crónica, la hipertensión arterial y la diabetes mellitus en Colombia - 2018 [Internet]. Colombia: Fondo Colombiano de Enfermedades de Alto Costo; 2018. Disponible en: https://cuentadealtocosto.org/site/publicaciones/situacion-de-la-enfermedad-renal-cronica-la-hipertension-arterial-y-la-diabetes-mellitus-en-colombia-2018/

2. $\mathrm{K} / \mathrm{DOQ}$ clinical practice guidelines for chronic kidney disease: evaluation, classification, and stratification. Am J kidney Dis Off J Natl Kidney Found. 2002;39(2 Suppl 1):S1-266.

3. Wolfe RA, Ashby VB, Milford EL, Ojo AO, Ettenger RE, Agodoa LY, et al. Comparison of mortality in all patients on dialysis, patients on dialysis awaiting transplantation, and recipients of a first cadaveric transplant. N Engl J Med. 1999;341(23):1725-30.

4. Ojo AO, Hanson JA, Meier-Kriesche H-U, Okechukwu CN, Wolfe RA, Leichtman $A B$, et al. Survival in recipients of marginal cadaveric donor kidneys compared with other recipients and wait-listed transplant candidates. J Am Soc Nephrol. 2001;12(3):589-97.

5. Costa-Requena G, Cantarell Aixendri MC, Rodriguez Urrutia A, Seron Micas D. Calidad de vida relacionada con la salud y trasplante renal: comparación con los valores poblacionales a los 6 meses postrasplante. Med Clin (Barc). 2014;142(9):393-6.

6. Daga Ruiz D, Fernández Aguirre C, Segura González F, Carballo Ruiz M. Indicaciones y resultados a largo plazo de los trasplantes de órganos sólidos: Calidad de vida en pacientes trasplantados. Med Intensiva. 2008;32:296-303.

7. Nino-Murcia A, Pinto Ramirez JL, Nino-Torres L. Organ transplantation in Colombia. Transplantation. 2018;102(11):1779-82.

8. Metzger R, Delmonico FL, Feng S, Port FK, Wynn JJ, Merion RM. Expanded criteria donors for kidney transplantation. Am J Transplant. 2003;3(Suppl 4):114-25.

9. Bajwa M, Cho YW, Pham PT, Shah T, Danovitch G, Wilkinson A, et al. Donor biopsy and kidney transplant outcomes: An analysis using the Organ Procurement and Transplantation Network/United Network for Organ Sharing (OPTN/UNOS) Database. Transplantation. 2007; 84(11):1399-405.

10. Valjalo R, Reynolds E, Herrera P, Espinoza A, González F. Resultados a largo plazo en trasplante renal de donantes con criterios expandidos. Rev Med Chile. 2016;144:22-9.

11. Pascual J, Zamora J, Pirsch JD. A systematic review of kidney transplantation from expanded criteria donors. Am J Kidney Dis. 2008;52(3):553-86.

12. García-Padilla PK, Vargas-Brochero MJ, Hurtado-Uriarte M, González-González CA, Rodríguez-Sánchez MP, Patiño-Trejos JA, et al. Caracterización de trasplantados renales con donantes de criterios expandidos. Acta Médica Colombiana. 2019;44(3):1-5.

13. Instituto Nacional de Salud. Informe anual Red de Donación y Trasplantes 2018. Bogotá D.C.: Instituto Nacional de Salud; 2018. 
14. Haas M, Sis B, Racusen LC, Solez K, Glotz D, Colvin RB, et al. Banf 2013 meeting report: Inclusion of C4d-negative antibody-mediated rejection and antibody-associated arterial lesions. Am J Transplant. 2014; 14(2):272-83.

15. Loupy A, Haas M, Solez K, Racusen L, Glotz D, Seron D, et al. The Banff 2015 Kidney Meeting Report: Current challenges in rejection classification and prospects for adopting molecular pathology. Am J Transplant. 2017;17(1):28-41.

16. Rocio Arias Y, Salinas Nova MA, Ivo Montaño J. Criterios de asignación para trasplante renal en Colombia [Internet]. Colombia: Instituto Nacional de Salud; 2018. Disponible en: https://www.ins.gov.co/Direcciones/RedesSaludPublica/DonacionOrganosYTejidos/DocumentosTecnicos/Consenso $\% 20$ Criterios $\% 20$ de $\% 20$ Asignaci $\%$ C3 $\%$ B3n $\% 20$ Trasplante $\% 20$ Renal\%20en\%20Colombia.pdf

17. Bozkurt B, Kılıç M. Marginal donors in renal transplantation. Transplant Proc. 2015;47(5):1273-81.

18. Filiopoulos V, Boletis JN. Renal transplantation with expanded criteria donors: Which is the optimal immunosuppression? World J Transplant. 2016;6(1):103-14

19. Foroutan F, Friesen EL, Clark KE, Motaghi S, Zyla R, Lee Y, et al. Risk factors for 1-year graft loss after kidney transplantation: Systematic review and meta-analysis. Clin J Am Soc Nephrol. 2019;14(11):1642-50.

20. Coemans M, Süsal C, Döhler B, Anglicheau D, Giral M, Bestard O, et al. Analyses of the short- and long-term graft survival after kidney transplantation in Europe between 1986 and 2015. Kidney Int. 2018:94(5):964-73.

21. Prommool S, Jhangri GS, Cockfield SM, Halloran PF. Time dependency of factors affecting renal allograft survival. J Am Soc Nephrol. 2000;11(3):565-73.

22. Pinto Ramírez JL. Factores asociados a la pérdida del injerto renal mortalidad en Colombiana de Trasplantes entre el 2008 al 2019 [tesis de maestría en internet]. [Bogotá, Colombia]: Universidad del Rosario Escuela Medicina y Ciencias de la Salud; 17/06/2020. Disponible en: https://repository.urosario.edu.co/handle/10336/25254

23. Rodríguez VM, Parra Morales M, Rocha Ortiz S, Castro Salas M, Villalobos Sepúlveda C. Supervivencia del injerto y del receptor en el trasplante renal en el hospital Guillermo Gran Benavente (Concepción, Chile). Enferm Nefrol. 2014;14(4):292-8.
24. Ticona-Garrón $A B$, Álvarez-Rangel LE, Jiménez-Domínguez $A$, Cruz-Santiago J, Medina-Uicab C, Meza-Jiménez G, et al. Supervivencia del paciente e injerto renal al año de trasplante de donante fallecido; comparación con resultados de donante vivo. Rev Mex Urol. 2010;70(6):347-53.

25. Nga HS, Andrade LGM, Contti MM, Valiatti MF, Silva MM da, Takase HM. Evaluation of the 1000 renal transplants carried out at the University Hospital of the Botucatu Medical School (HCFMB) - UNESP and their evolution over the years. Brazilian J Nephrol. 2018;40:162-9.

26. Koukoulaki M, Kyriakopoulos G, Nikolaidis C, Balaska A, Pistolas D, Drakopoulos S. Proposal of categorization of expanded criteria donors in renal transplantation. Transplant Proc. 2014;46(9):3168-71.

27. Martínez-Vaquera S, Navarro Cabello MD, López-Andreu M, Jurado JMD Haad CR, Salas RO, et al. Outcomes in renal transplantation with expanded-criteria donors. Transplant Proc. 2013;45(10):3595-8.

28. Palkoci $B$, Vojtko M, Fialová J, Osinová $D$, Lajčiaková M. Results of kidney transplantation from expanded criteria donors: A single-center experience. Int J Organ Transplant Med. 2018;9(1):1-9.

29. Pascual J, Pérez-Sáez MJ. El Kidney Donor Profile Index: ¿se puede extrapolar a nuestro entorno? Nefrología (Madrid). 2016;36:465-8.

30. Ramalho ALAS, Custodio FB, Tokuda BM, Caldas HC, Fernandes IM, Abbud-Filho $\mathrm{M}$, et al. Clinical and histopathologic comparative analysis between kidney transplant recipients from expanded-criteria donors and standard-criteria donors. Transplant Proc. 2013;45(9):3234-8.

31. De Rosa P, Muscogiuri G, Sarno G. Expanded criteria donors in kidney transplantation: The role of older donors in a setting of older recipients. ISRN Transplantation. 2013;2013:301025.

32. Aubert O, Kamar N, Vernerey D, Viglietti D, Martinez F, Duong-VanHuyen J-P, et al. Long term outcomes of transplantation using kidneys from expanded criteria donors: prospective, population based cohort study. BMJ. 2015:351:h3557.

33. Meier-Kriesche HU, Schold JD, Srinivas TR, Kaplan B. Lack of improvement in renal allograft survival despite a marked decrease in acute rejection rates over the most recent era. Am J Transplant. 2004;4(3):378-83. 